\title{
BMJ Open Randomised controlled trial examining the effect of an outpatient exercise training programme on haemodynamics and cardiac MR parameters of right ventricular function in patients with pulmonary arterial hypertension: the ExPAH study protocol
}

\author{
Karen S W Chia, ${ }^{1,2}$ Steven G Faux, ${ }^{3,4,5}$ Peter K K Wong, ${ }^{1,2,6}$ Cameron Holloway, ${ }^{4,5}$ \\ Hassan Assareh, ${ }^{1,7}$ Craig S McLachlan, ${ }^{1}$ Eugene Kotlyar ${ }^{4,5}$
}

\begin{abstract}
To cite: Chia KSW, Faux SG Wong PKK, et al.

Randomised controlled trial examining the effect of an outpatient exercise training programme on

haemodynamics and cardiac MR parameters of right ventricular function in patients with pulmonary arterial hypertension: the ExPAH study protocol. BMJ Open 2017;7:e014037. doi:10.1136/bmjopen-2016014037
\end{abstract}

- Prepublication history for this paper is available online. To view these files please visit the journal online (http://dx.doi.org/10.1136/ bmjopen-2016-014037).

Received 25 August 2016 Revised 9 November 2016 Accepted 5 January 2017

\section{CrossMark}

For numbered affiliations see end of article.

Correspondence to Dr Karen SW Chia; Karen.chia@me.com

\section{ABSTRACT}

Introduction: Pulmonary hypertension $(\mathrm{PH})$ is a potentially life-threatening condition characterised by elevated pulmonary artery pressure. Early stage PH patients are often asymptomatic. Disease progression is associated with impairment of right ventricular function and progressive dyspnoea. Current guidelines recommend exercise training (grade lla, level B). However, many questions remain regarding the mechanisms of improvement, intensity of supervision and optimal frequency, duration and intensity of exercise. This study will assess the effect of an outpatient rehabilitation programme on haemodynamics and cardiac right ventricular function in patients with pulmonary arterial hypertension (PAH), a subgroup of $\mathrm{PH}$.

Methods and analysis: This randomised controlled trial involves both a major urban tertiary and smaller regional hospital in New South Wales, Australia. The intervention will compare an outpatient rehabilitation programme with a control group (home exercise programme). Participants will be stable on oral PAHspecific therapy. The primary outcome measure will be right ventricular ejection fraction measured by cardiac MRI. Secondary outcomes will include haemodynamics measured by right heart catheterisation, endurance, functional capacity, health-related quality of life questionnaires and biomarkers of cardiac function and inflammation.

Ethics approval and dissemination: Ethical approval has been granted by St Vincent's Hospital, Sydney (HREC/14/SVH/341). Results of this study will be disseminated through presentation at scientific conferences and in scientific journals.

Trial registration number: ACTRN12615001041549; pre-results.

\section{Strengths and limitations of this study}

- To the best of our knowledge, this is the first clinical trial evaluating the effect of an outpatient rehabilitation programme (OP REH) on right ventricular ejection fraction measured by cardiac MRI.

- We will also be measuring the effect of an OP REH on haemodynamic parameters as assessed by right heart catheterisation.

- The nature of the OP REH reflects 'real-world' conditions generalisable to routine clinical practice (1 hour, twice weekly, for 12 weeks).

- Although participants are not blinded to their allocated treatment group, all study personnel are blinded.

- The short follow-up period for this study (6 months) is a limitation; however, we were limited by budget constraints.

\section{INTRODUCTION}

Pulmonary hypertension (PH) is a condition characterised by elevated pulmonary arterial pressure (PAP), and is associated with fatigue, syncope, angina and progressive exertional dyspnoea. It is defined as a mean resting pulmonary arterial pressure (mPAP) of $\geq 25 \mathrm{~mm} \mathrm{Hg}$, measured by right heart catheterisation (RHC). ${ }^{1} \mathrm{PH}$ is characterised by vascular remodelling, with a consequent increase in PAP, resulting in increased right ventricular afterload. ${ }^{2}{ }^{3}$ The ability of the right ventricle to adapt to the increased right-sided afterload is key to prognosis. ${ }^{3}{ }^{4}$ 
Patients with PH generally have a poor prognosis ${ }^{5}$ in particular patients with pulmonary arterial hypertension (PAH). Median survival of patients with PAH is between 2 and 3 years. ${ }^{6}$ Underlying aetiology is an important prognostic factor, with scleroderma-related PAH (a subtype of $\mathrm{PAH}$ ) having a worse prognosis. ${ }^{7-9}$

$\mathrm{PAH}$ is a precapillary subtype of $\mathrm{PH}$, with additional haemodynamic criteria of pulmonary artery wedge pressure $\leq 15 \mathrm{~mm} \mathrm{Hg}$ and pulmonary vascular resistance (PVR) $>3$ Wood units. ${ }^{10}$ The focus of this study is patients with PAH.

Exercise has not previously been recommended in patients with $\mathrm{PAH}$, particularly in patients with more advanced disease, due to safety concerns. ${ }^{11}$ However, exercise training has been shown to be safe in patients with $\mathrm{PAH},{ }^{12}$ and current European guidelines recommend exercise training and rehabilitation in experienced centres for patients with PAH who are clinically stable, on optimal pharmacological treatment (grade IIa, level B). ${ }^{1}$

The first major study involving patients with $\mathrm{PH}$ (patients with $\mathrm{PAH}$ and chronic thromboembolic $\mathrm{PH}$ ) showed that exercise was beneficial. Thirty participants were randomised to either exercise training or a control group (education on healthy nutrition, muscular relaxation). ${ }^{13}$ The exercise training group underwent an intensive 3-week inpatient programme of a minimum of 2 hours/day of exercise (strength and endurance training, and respiratory muscle exercises) and education (healthy nutrition, muscle relaxation). This was followed by a 12-week home exercise programme (HEP). The exercise training group had a marked improvement in 6 min walk test (6MWT) distance compared with the control group, with mean between-group difference of $111 \mathrm{~m}(95 \%$ CI 65 to $139 ; \mathrm{p}<0.001)$. Quality of life (QOL), measured by the Short Form-36 (SF-36), ${ }^{14}$ also improved in the exercise training group.

Seventeen more studies examining the effect of exercise training on $\mathrm{PH}$ have been published up to June 2016, since the above landmark study in 2006. These studies are generally small in size, and are of varying quality (only six randomised controlled trials (RCTs)). ${ }^{15}$ Several studies ${ }^{12}{ }^{16-22}$ using the Mereles et $a l^{13}$ inpatient protocol (described previously) reported improvement in a number of outcome measures, including 6MWT and QOL. The largest RCT of these studies $(n=87)$, recently published by Ehlken $e t a l^{20}$ has further added to the current body of knowledge by showing that exercise training using the Mereles et al inpatient protocol improved haemodynamics.

However, it is difficult to replicate the intensity of this inpatient exercise programme in routine clinical practice due to limited resources and personnel. In the Australian context, the Mereles et al protocol is not currently widely available due to cost constraints. Cardiac and pulmonary rehabilitation programmes in Australia are usually run by public hospital outpatient services or community settings, two to three times per week at minimal or no cost to patients. ${ }^{23} 24$
A more achievable programme of exercising two to three times per week for 1 hour per session may still have beneficial effects on walk distance, ${ }^{25-30}$ peak oxygen consumption, ${ }^{26}$ QOL, self-reported symptoms, ${ }^{27}$ fatigue and activity levels. ${ }^{28}$ We have therefore designed this study with 'real-world' conditions in mind to ensure broad generalisability. Given anxiety and depression are important factors associated with dyspnoea, ${ }^{31}$ we have chosen to include a structured psychology intervention as part of the outpatient rehabilitation programme (OP REH) to address this.

\section{Hypotheses}

We hypothesise that an OP REH (involving endurance training, respiratory muscle training (RMT) and structured psychology intervention) will have beneficial effects on cardiac remodelling (reflected in an improvement in right ventricular ejection fraction (RVEF)), haemodynamics, strength and endurance, and QOL. We hypothesise that these benefits will be due to improved right-sided cardiac function as determined by an improvement in RVEF.

The key difference of this study compared with previous studies is the inclusion of cardiac MRI (cMRI) and haemodynamics, as well as other secondary outcome measures (as listed below) that will help elucidate the mechanism of improvement of exercise training. If secondary outcome measures increase despite no improvement in RVEF, this would suggest that other mechanisms are responsible for the improvement in exercise capacity.

\section{Objectives}

To assess whether the 'real-world' frequency of OP REH available in Australia (ie, 1 hour, twice weekly, for 12 weeks) has a beneficial effect on:

1. Cardiac function (RVEF and volume as assessed by cMRI);

2. Haemodynamics, measured by RHC, the gold standard for assessing $\mathrm{PAH} ;{ }^{10}$

3. Dominant hand grip strength (DHGS) and exercise capacity (6MWT);

4. Function and QOL (measured by questionnaires: the Cambridge Pulmonary Hypertension Outcome Review (CAMPHOR), Depression and Anxiety Severity Scale (DASS21), Lawton's Instrumental Activities of Daily Living (IADL);

5. Serum biomarkers of cardiac function and inflammation ( $\mathrm{N}$ terminal pro b-type natriuretic peptide (NT-proBNP), interleukin (IL-6));

6. Lung function (vital capacity).

\section{METHODS AND ANALYSIS}

\section{Study design}

The study is designed as a dual-centre, investigatorblinded clinical trial to compare the efficacy of an OP REH versus a HEP. Participants will be randomised in a 1:1 ratio. The exercise intervention will occur for 12 weeks. 


\section{Study population}

Study participants will be identified by cardiologists and rheumatologists during the course of routine care at St Vincent's Hospital, Sydney, Australia, and from the Coffs Harbour PAH Clinic, an outpatient clinic affiliated with the Coffs Harbour Health Campus (regional hospital). Participants must fulfil all inclusion criteria (table 1). Exclusion criteria are outlined in table 2. We have excluded WHO Class IV participants, due to safety concerns regarding exercising these participants in an outpatient setting.

\section{Recruitment, randomisation and allocation concealment}

The ExPAH study has been designed in line with the Consolidated Standards of Reporting Trials (CONSORT) statement. ${ }^{33}$ The study protocol is outlined in figure 1.

All eligible participants will have the aims, methods and potential adverse events explained to them by the study investigators prior to signing informed consent. Recruitment will occur until sample size requirements are met (anticipated duration of recruitment 1-2 years).

Randomisation will occur after screening (see below) and will be carried out using a minimisation scheme to achieve balance by research site and presence of connective tissue disease-associated $\mathrm{PAH}$, as this particular cohort of patients with PAH has a worse prognosis ${ }^{7}$ as mentioned previously. Randomisation will be carried out by an independent biostatistician using a customised programme for minimisation (QMinim) ${ }^{34}$ The participant will be allocated to either control (HEP) or the intervention (OP REH). Administrative staff not involved with the project will inform participants via mail regarding their allocation group.

Participants will not be blinded to the intervention. However, assessments and data analysis will be carried out by blinded investigators.

\begin{tabular}{ll} 
Table 1 & Inclusion criteria \\
\hline Criteria & Characteristics of eligible participants \\
\hline 1. & $\begin{array}{l}\text { Male and female patients aged } \geq 18 \text { years } \\
2 .\end{array}$ \\
& $\begin{array}{l}\text { PAH diagnosed by RHC (at any time prior to } \\
\text { baseline) with the following parameters: }\end{array}$ \\
i. $\quad$ Resting $\mathrm{mPAP} \geq 25 \mathrm{~mm} \mathrm{Hg}$, and \\
3. & $\begin{array}{l}\text { ii. } \quad \text { Resting PCWP or LVEDP } \leq 15 \mathrm{~mm} \mathrm{Hg} \\
\text { preceding } 3 \text { months }\end{array}$ \\
4. & $\begin{array}{l}\text { Female participants of childbearing potential } \\
\text { must have a negative pregnancy test and } \\
\text { agree to use contraception for study } \\
\text { duration. }\end{array}$ \\
\end{tabular}

LVEDP, left ventricular end diastolic pressure; mPAP, mean pulmonary arterial pressure; PCWP, pulmonary capillary wedge pressure; PAH, pulmonary arterial hypertension; PVR, pulmonary vascular resistance; $\mathrm{RHC}$, right heart catheterisation.
Outcome measures

Cardiac MRI

cMRI was chosen as it is the 'gold standard' for assessing right ventricular structure and function. ${ }^{35}$ cMRI studies will be performed using a single 1.5 Tesla (T) MR system (Philips) and 3T (Siemens Healthcare, Germany). A complete stack of short axis images will be obtained during breath-hold and cardiac gating for cine, and late gadolinium enhancement (LGE) imaging. LGE imaging will be performed as previously described ${ }^{36}$ using a T1-weighted phase-sensitive inversion recovery sequence $\sim 8 \mathrm{~min}$ after intravenous administration of a contrast agent (Gadoterate meglumine-Gd-DOTA, Dotarem, Guerbet LLC, France; $0.15 \mathrm{mmol} / \mathrm{kg}$ body weight). Imaging parameters will be the same as previously published. ${ }^{37}$ All cMRIs will be analysed using

Table 2 Exclusion criteria for the ExPAH study

Criteria Characteristics of ineligible participants

1. General exclusion criteria:

- Pregnancy

- Presence of cognitive impairment and/or neuromuscular weakness of sufficient severity to prevent participation in exercise classes

- Non-primary English language limiting ability to comply with exercise classes

- WHO Functional Class IV

- enrolled in a formal exercise training programme within preceding 12 weeks

2. PAH-specific:

- Change in PAH medication within preceding 3 months

3. Presence of significant cardiac disease, including:

- Unstable angina pectoris (ie, chest pain not controlled with medications)

- Unstable CCF requiring escalation of medication within preceding 3 months

- Exercise-induced arrhythmias such as atrial fibrillation, ventricular tachycardia

- Hypertrophic obstructive cardiomyopathy

4. Patients unable to undergo MRI:

- Presence of impaired renal function (eGFR $<60 \mathrm{~mL} / \mathrm{min}$ ) due to a possible adverse event with intravenous gadolinium contrast

- In situ pacemaker or implantable cardiac defibrillator

- Other metal or electronic implants affected by a magnetic field

- Shrapnel injuries

- Previous eye injury involving metal fragments

- Metal clips in cerebral blood vessels

- Joint replacement within 3 months

- Previous serious adverse event to MRI gadolinium contrast agents

CCF, congestive cardiac failure; eGFR, estimated glomerular filtration rate; $\mathrm{PAH}$, pulmonary arterial hypertension. 
Figure 1 ExPAH protocol: enrolment, allocation, follow-up and analysis. Participants are screened for eligibility. If eligible, once informed consent obtained, participants are then randomised to control group (home exercise) or intervention group (outpatient rehabilitation exercise programme). Participants are reviewed at weeks 12 and 26; participants who have not completed the required attendance (>70\%) are recorded. Analysis of results will be both by intention-to-treat and per protocol methods. OP REH, outpatient rehabilitation programme.

\section{Enrolment \\ Screened for eligibility}

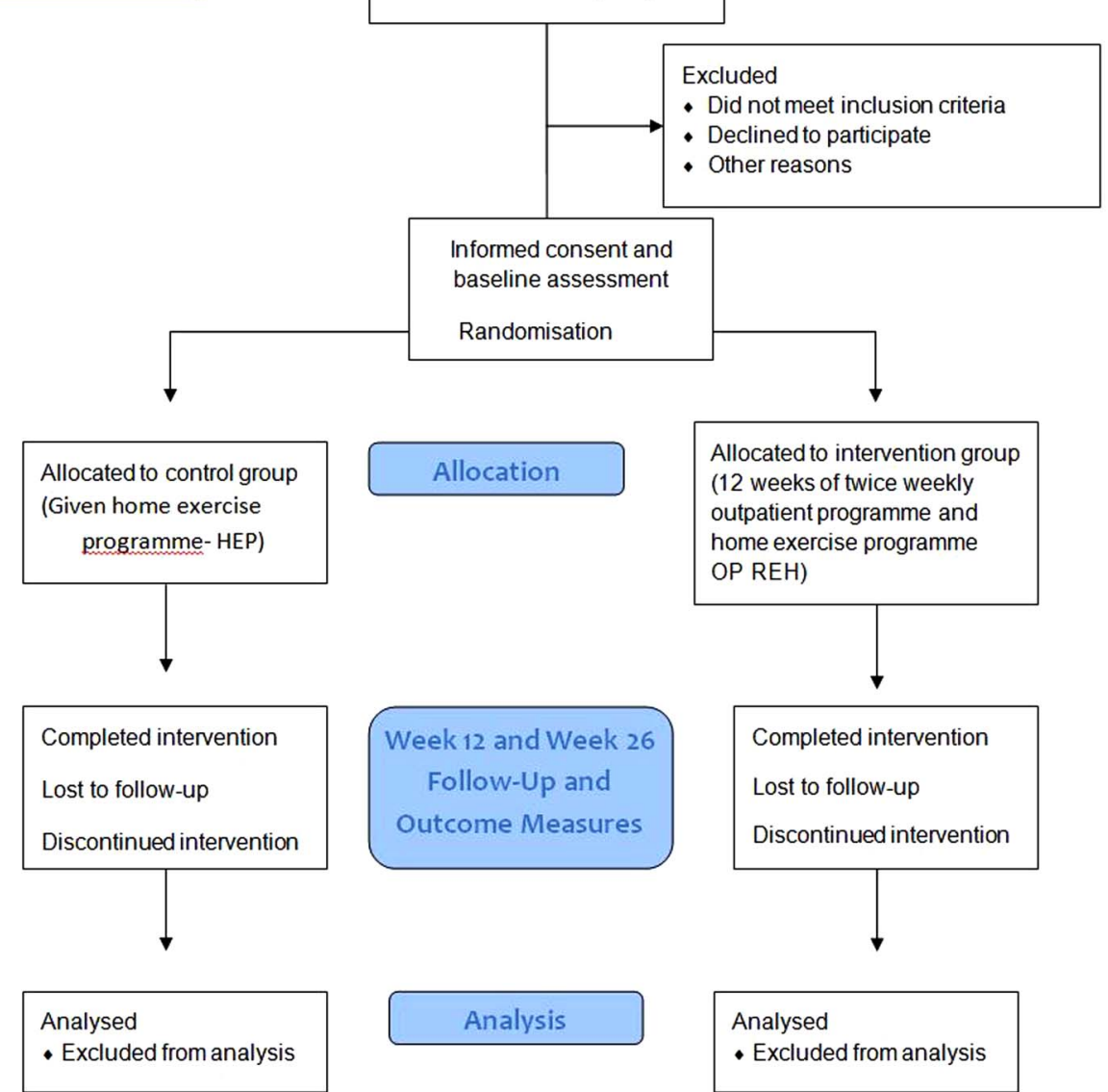

CMR42 software (Canada), as previously described, ${ }^{38}$ by the investigators blinded to participant randomisation group.

\section{Right heart catheterisation}

Standard haemodynamic parameters measured by $\mathrm{RHC}^{10}$ were chosen as a secondary outcome measure based on the known impairments in $\mathrm{PAH}$ and previous noted improvements with inpatient rehabilitation in the cardiac index, mPAP and PVR. ${ }^{20}$ RHC will be performed according to a standard protocol ${ }^{10}$ by trained cardiologists who are blinded to the participant's randomisation group.

\section{Frailty and exercise capacity}

As skeletal muscle weakness is a known complication of $\mathrm{PH},{ }^{39-43}$ DHGS will also be included as a secondary end point. In addition, decreased hand grip strength (HGS) has been noted to be associated with increased all-cause mortality. ${ }^{44}$ DHGS will be measured by a physiotherapist blinded to the participant's randomisation group (using a Jamar Hydraulic Hand Dynamometer, Sammons Preston, Canada).

Exercise capacity, as measured by 6MWT, has been widely used as an outcome measure in trials of exercise training and pharmacological management of PAH. This study will assess whether the increase in 6MWT seen with previous trials ${ }^{45} 46$ will be able to be reproduced with an outpatient programme. Exercise capacity will be measured by a trained assessor (who is blinded to the study randomisation). All participants will be assessed undertaking two supervised 6MWTs separated by a rest of at least $30 \mathrm{~min}$, in accordance with European Respiratory Society/American Thoracic Society technical standards. ${ }^{47}$

\section{Questionnaires of function and QOL}

Mental health disorders are very common, with $\sim 35 \%$ of patients with PAH suffering depression and anxiety. ${ }^{48}$ Depression and anxiety in patients with PAH is associated with reduced QOL. ${ }^{49}$ While reduction in health-related QOL is well documented in $\mathrm{PAH}$, the focus of these studies have been on physical function, mental health and social isolation, ${ }^{50}$ with less emphasis on activities of daily living. We have therefore included a short questionnaire of daily activities (Lawton's IADL) which looks at other household tasks/roles. ${ }^{51}$ All study participants will complete three questionnaires at baseline, 12 and 26 weeks:

I. The CAMPHOR ${ }^{52}-\mathrm{a}$ PH-specific QOL outcome measure validated in the Australian population; ${ }^{53}$

II. Lawton's IADL ${ }^{54}$ - a measure of impairment of daily activities;

III. DASS ${ }^{55}$ - to assess concurrent depression and anxiety. 
Blood test markers of cardiac function and inflammation

N-terminal fragment (NT-proBNP) and IL-6 were included as outcome measures for their prognostic value in $\mathrm{PH}^{56}{ }^{57} \mathrm{NT}$-proBNP is the inactive N-terminal component produced when the ventricles secrete a prohormone, pro-BNP that is cleaved into an active form (BNP) and an inactive fragment (NT-proBNP) ${ }^{58}$ NT-proBNP is a clinically useful and minimally invasive test that correlates well with survival. NT-proBNP values $>1400$ to 1700 were associated with a poorer prognosis. ${ }^{59}$ IL-6 is a marker of inflammation that adds further prognostic information in patients with $\mathrm{PAH}^{57}$ Blood samples will be collected at baseline, weeks 12 and 26 follow-up visits in serum separator tubes for analysis.

\section{Lung function}

Respiratory muscle weakness is a known association with PAH ${ }^{60}{ }^{61}$ A previous study of inspiratory muscle training (IMT) in patients with PAH has demonstrated significant improvements in respiratory muscle strength, functional capacity, and dyspnoea and fatigue perception. ${ }^{30}$ Vital capacity is useful for monitoring respiratory muscle weakness. ${ }^{62}$ Therefore, all participants will undergo vital capacity testing to ascertain whether OP REH improves respiratory muscle weakness. We did not include direct measures of respiratory muscle strength (maximum inspiratory pressure and maximum expiratory pressure) due to financial constraints and safety concerns in this population.

\section{Assessment}

All participants will undergo the following assessments:

\section{The medical screening visit}

Participants will have eligibility reviewed, demographics (age, gender, aetiology of PAH), WHO Functional PH Class, medical history, physical examination and informed consent (if eligible) documented. Eligible participants will undergo cMRI, RHC, lung function tests (vital capacity) and venepuncture for cardiac markers (NT-proBNP and IL-6). Candidates who fail to meet inclusion criteria, or fulfil exclusion criteria will have the reason for study ineligibility documented.

\section{Baseline visit}

Participants will return for the baseline visit to review results. Participants will also have a separate visit with a trained assessor (who is blinded to the study randomisation) to undergo two supervised 6MWTs separated by a rest of at least $30 \mathrm{~min}$, in accordance with international guidelines. ${ }^{47}$ DHGS will be recorded, and participants will complete QOL and function questionnaires as described previously. Participants will then be randomised as outlined above.

\section{Visit 1 (week 12-14)}

Participants will undergo medical review including physical examination at completion of the intervention period after week 12. Adverse events will be recorded, and any clinical episodes (change in PAH-specific medication or hospitalisation secondary to $\mathrm{PAH}$ ) will be documented. Participants will undertake two supervised 6MWTs by a blinded assessor, have DHGS measured and complete the three study questionnaires, as before. Participants will also undergo cMRI, RHC, lung function tests and venepuncture for cardiac markers (NT-proBNP and IL-6).

\section{Visit 2 (week 26)}

Participants will undergo medical review, including physical examination. Any adverse events will be recorded, and any clinical episodes (change in PAH-specific medication or hospitalisation secondary to $\mathrm{PAH}$ ) will be documented. Participants will undertake two supervised 6MWTs by a blinded assessor as before. Participants will undergo venepuncture for serum cardiac and inflammatory biomarkers. Participants will have assessment of DHGS and complete the three QOL questionnaires as in visit 1 .

\section{Interventions}

Exercise:

\section{Intervention (OP REH)}

All participants randomised to this group will undertake physiotherapist-supervised, outpatient hospital based, group exercise training for 1 hour/week, twice weekly, for 12 weeks, with the following components:

\section{Endurance training}

Twenty minutes of lower limb endurance training by walking or cycling. Participants will start at a speed of $60 \%$ of the speed of their 6MWT. If a participant chooses to cycle instead of walk, then intensity will be based on an initial level of light-to-somewhat hard exertion using the Borg Rating of Perceived Exertion (RPE) ${ }^{63}$ at a level of 12-13).

If participants are unable to complete $20 \mathrm{~min}$ of lower limb endurance training, they will increase duration by $5 \mathrm{~min}$ each time until able to complete 20 min total. During the exercise training class, participants' heart rate (HR) will be monitored via portable HR and oxygen saturations machine. HR will be kept $<120 \mathrm{bpm}$, as per current recommendations. ${ }^{64}$ Participants' oxygen saturations $\left(\mathrm{O}_{2}\right.$ sats $)$ will be kept $>88 \%$ on room air, as per recent international guidelines. $^{65}$

Progression of training will occur by increasing the treadmill gradient by $1 \%$ on each visit, or increase speed by $5 \%$ each week, as tolerated, maintaining an intensity of 12-13 on Borg RPE, if HR and $\mathrm{O}_{2}$ sats are able to be maintained within the range as stated above.

2. Respiratory muscle training

During the exercise training group sessions, all participants will undergo RMT taught by a physiotherapist. Training will involve education at the first 
intervention visit regarding breathing techniques to manage symptoms (pursed-lip breathing and diaphragmatic breathing). All subsequent sessions will include $10 \mathrm{~min}$ of supervised IMT using individual IMT devices (threshold IMT positive inspiratory pressure device, Phillips). Ideally, maximal inspiratory pressure (MIP) should be measured by formal lung function tests. However, these tests are expensive, and may be difficult for some patients to undertake. Even without formal MIP, IMT training can be prescribed, using the Borg RPE at a target of RPE 12-13. ${ }^{66}$ The benefit from IMT is seen at levels as low as $30 \%$ of MIP. $^{67}$ Training at a Borg RPE of 12-13 correlates with $\sim 40 \%$ of MIP. $^{66}$

3. Strength training

To achieve benefit, strength training needs to occur at repetitions of $50-80 \%$ of the one repetition maximum (1RM), at least two to three times per week. ${ }^{65}$ If the $1 \mathrm{RM}$ is difficult to obtain, we will alternatively use a weight that the participant can only lift 10 times. ${ }^{23}$ Strength training of the upper limbs and lower limbs will occur twice weekly at the supervised training sessions for a total of $20 \mathrm{~min}$. Initial intensity will be based on the 1RM, and increased by $10 \%$ of load once the participant is able to perform two sets of 10 repetitions on two consecutive training sessions. ${ }^{68}$ Upper limb exercises will include dumbbell row, seated chest press and lateral arm raise.

Lower limb strength training exercises will include step ups and sit to stands.

\section{HEP at week 3}

Participants will be given a HEP, consisting of instructions to walk 3 days/week for $30 \mathrm{~min}$ total per day, in addition to attending the twice weekly classes with the physiotherapist. Participants will also be provided with their own IMT device as described above, and given written instructions on undertaking IMT training for $30 \mathrm{~min}$ per day for 5 days/week. Participants who desaturate to $<88 \%$ on room air (RA) while exercising (as determined during their exercise class) will be given a modified HEP of interval training. This will consist of advice to walk for $1 \mathrm{~min}$ then $1 \mathrm{~min}$ of rest, initially for 15-20 min for the first four sessions, then increasing to $45-60 \mathrm{~min}$ (including rest time). ${ }^{69}$ All participants will be advised to cease their exercise programme until review by their physiotherapist if they become symptomatic (feel unwell, dizziness, light headedness, dyspnoea).

\section{Control group (HEP)}

Participants randomised to the HEP will be given written material recommending a walking programme for 5 days/week for $30 \mathrm{~min}$ total per day. This can be broken up into smaller sessions per day to make it more achievable (eg, walking for $10 \mathrm{~min}$, three times per day). Advice will be provided on how to stay motivated, as well as a diary to record exercise undertaken.
Psychology intervention

Intervention group (OP REH)

The OP REH group will also have standardised individual counselling support for three sessions $(20 \mathrm{~min}$ sessions) via telephone by the study psychologist during the 12-week intervention. This will be structured as follows:

Session 1: education on how to manage hyperventilation and anxiety during breathlessness. ${ }^{70}$

Session 2: structured cognitive-behavioural therapy to manage dyspnoea. This will involve education and challenging of catastrophic thoughts. ${ }^{71}$

Session 3: skills training. This will involve education and practice in progressive muscle relaxation. ${ }^{72}$ Participants will be given written instructions (as well as verbal instructions) regarding how to do this.

\section{Control group}

The control group will not receive any psychology input as part of the study protocol.

\section{Adherence}

The intervention group will have attendance at each exercise session recorded by the treating physiotherapist. The intervention and the control groups will both be given a diary to record their home activity level. Participants who have completed $>70 \%$ of sessions (including self-documented HEP) will be deemed to have completed the programme.

\section{Withdrawal criteria}

Participants may voluntarily withdraw from the study at any time, or if they experience a serious adverse event (SAE) that is associated with an exercise session (during or up to 24 hours postintervention). An SAE is defined as an untoward medical occurrence that results in any of the following: death, a life-threatening event, inpatient hospitalisation, persistent or significant disability/incapacity, and a condition requiring medical or surgical intervention. Other adverse events (defined as any unfavourable and unintended sign, symptom or disease temporally associated with any of the investigations or the intervention), such as failure to progress according to protocol, syncope and presyncope will also be recorded. Participants may also be withdrawn if the designated blinded medical assessor at the participant's site deems it unsafe for them to continue with the study.

\section{Data collection, confidentiality, storage and archiving of study documents}

All personnel involved in data collection will be blinded. Study documents will be de-identified and stored for 15 years, as per protocol for non-clinical trial notification (CTN) interventional studies. ${ }^{73}$ Data will be stored electronically, on double password-protected computers. Data on paper (the Clinical Research File) will be stored in a locked, secure office. The principal investigators will have access to the final trial data set. 


\section{Analysis of outcome measures}

The primary outcome measure is the absolute change (increase) in RVEF from baseline to visit 1, assessed by cMRI.

Secondary outcome measures include:

A. Absolute change in other parameters on cMRI from baseline to visit 1 . These parameters include stroke volume, right ventricular end systolic volume, right ventricular end diastolic volume, right ventricular mass, left ventricular end systolic volume, left ventricular end diastolic volume and left ventricular mass, cross-sectional septum thickness at the level of the papillary muscle.

B. Absolute change in haemodynamics measured by RHC from baseline to visit 1, including PVR, cardiac output, mean right atrial pressure, mPAP and systemic vascular resistance.

C. Absolute change in measures of strength and exercise capacity from baseline to visit 1 and 2, including 6MWT, muscle strength testing (DHGS).

D. Measures of function and QOL scores at baseline, visit 1 and 2, including the CAMPHOR, Lawton's IADL and DASS21.

E. Blood test markers of cardiac function and inflammation (NT-proBNP and IL-6).

F. Lung function tests (vital capacity).

\section{Statistical considerations}

Sample size calculation

Power calculations are based on comparison of randomised arms achieving the primary outcome of an increase in RVEF. We consider a 5\% absolute excess increase in RVEF, measured by cMRI, for the intervention group compared with the control group as the desired clinically important effect to be detected. ${ }^{74}$ This increase corresponds to at least a $14 \%$ improved outcome for the intervention group, in conjunction with no improvement in the control group within a population of patients with an average RVEF of $35 \%{ }^{75}$

Although measurement of RVEF by cMRI has high reproducibility, ${ }^{76}$ we will minimise bias by conducting analyses of cMRI by the same investigator. ${ }^{75}{ }^{76}$ In our power calculation, we have incorporated 6\% SD for both SD of changes compared with baseline and measurement variability at the time of follow-up, reported by Addetia et $a l .{ }^{75}$ Using two-sided normal test with a significance level of $5 \%$ based on clinical equivalent design ${ }^{77}$ and potential $10 \%$ drop off, 26 patients in each arm are required to detect a 5\% excess increased RVEF associated with intervention versus control group with a power of $80 \%$. Our power calculation is comparable to other published papers calculating sample size required for cMRI and RVEF trials. ${ }^{62} 76$

\section{Analysis populations}

The primary and secondary end points A-D will be assessed in the intention-to-treat study population, defined as all participants who are randomised according to their randomised arm. In this analysis, participants missing their primary end point data for any reason will be imputed as failures for binary end points, and will have their previous observation carried forward for continuous end points. The primary end point will be analysed as per protocol analysis (this excludes participant data from the time at which they cease their randomised intervention). Secondary end points $E-G$ will be assessed in a per protocol population, using available data.

\section{Results analysis}

Results will be analysed using STATA V.13 (StataCorp), SAS (Cary, North Carolina, USA) or R V.3.2.2 (R Core Team, Austria). Primary outcome and participant continuous characteristics will be compared between two randomised arms using a t-test or Wilcoxon test, as appropriate. Binary end points and categorical characteristics will be compared using a $\chi^{2}$ or Fisher's exact test as appropriate. In case of differences in baseline characteristics across arms, adjusted characteristics effects on outcomes and their changes over time points will be evaluated using generalised linear models or non-parametric equivalents. Adherence to intervention will be described based on data in the participant diary. Participants will be deemed to have completed the intervention if their total (attendance and self-recorded home diary) adherence is $>70 \%$. For QOL questionnaire components with categorical cut-offs (the DASS21) the proportion of participants in each category at each visit will be compared by randomised arm. As the CAMPHOR and Lawton's IADL are ordinal scales, ordinal-based tests and regression modelling or nonparametric equivalents will be used.

\section{DISCUSSION}

To the best of our knowledge, the ExPAH study will be the first RCT to measure both haemodynamics and cMRI parameters. It is hypothesised that the data obtained will define reverse cardiac remodelling (defined by an increase in RVEF) as a mechanism that is important in the improvement observed in patients with PAH following exercise training.

Secondary outcome measures will also measure the effect of an OP REH on HGS and exercise capacity, QOL, depression, stress and anxiety, cardiac biomarkers and vital capacity. While these outcomes are all of clinical relevance, such a comprehensive data set requires significant time commitment by participants. This may limit recruitment, adherence and attendance.

\section{Pragmatic approach}

There was some debate regarding which exercise components should be included. However, we were determined to design an exercise intervention with broad generalisability within a 'real-world' setting of limited therapy time and resources. 
The results of this study will assist in guiding clinical practice regarding the type of exercise, duration and frequency that is of benefit in patients with PAH.

\section{Author affiliations}

${ }^{1}$ University of New South Wales Rural Clinical School, Coffs Harbour, New South Wales, Australia

${ }^{2}$ Coffs Harbour Health Campus, Coffs Harbour, New South Wales, Australia ${ }^{3}$ Sacred Heart Rehabilitation, St Vincent's Health, Coffs Harbour, New South Wales, Australia

${ }^{4}$ University of New South Wales, Coffs Harbour, New South Wales, Australia ${ }^{5}$ St Vincent's Hospital Sydney, Coffs Harbour, New South Wales, Australia ${ }^{6}$ Mid-North Coast Arthritis Clinic, Coffs Harbour, New South Wales, Australia ${ }^{7}$ Epidemiology and Health Analytics Department, Western Sydney Local Health District, Coffs Harbour, New South Wales, Australia

Acknowledgements The authors wish to thank Professor Stephen Kerr (Kirby Institute, New South Wales, Australia) for his assistance with the Statistics section.

Contributors KSWC conceived the study, coordinated its design and drafted the manuscript. SGF and EK had significant input into the study protocol. PKKW, CSM, SGF and EK read and were involved in critical appraisal and revision of the manuscript. $\mathrm{CH}$ provided MRI expertise. HA provided statistical expertise. All authors approved the final manuscript prior to submission.

Funding KSWC has received grants from the Mid-North Coast Local Health District and the Royal Australasian College of Physicians for this study. KSWC and EK have received an unrestricted educational grant from Actelion. Actelion have reviewed the manuscript. However, final content and design is exclusively retained by the authors.

Competing interests None declared.

Ethics approval St Vincent's Hospital Research and Ethics Committee.

Provenance and peer review Not commissioned; externally peer reviewed.

Open Access This is an Open Access article distributed in accordance with the Creative Commons Attribution Non Commercial (CC BY-NC 4.0) license, which permits others to distribute, remix, adapt, build upon this work noncommercially, and license their derivative works on different terms, provided the original work is properly cited and the use is non-commercial. See: http:// creativecommons.org/licenses/by-nc/4.0/

\section{REFERENCES}

1. Galiè N, Humbert M, Vachiery JL, et al. 2015 ESC/ERS guidelines for the diagnosis and treatment of pulmonary hypertension: the Joint Task Force for the diagnosis and treatment of pulmonary hypertension of the European Society of Cardiology (ESC) and the European Respiratory Society (ERS): endorsed by: association for European Paediatric and Congenital Cardiology (AEPC), International Society for Heart and Lung Transplantation (ISHLT) Eur Heart J 2016;37:67-119.

2. Howard LS. Prognostic factors in pulmonary arterial hypertension: assessing the course of the disease. Eur Respir Rev 2011;20:236-42.

3. Peacock AJ, Vonk Noordegraaf A. Cardiac magnetic resonance imaging in pulmonary arterial hypertension. Eur Respir Rev 2013;22:526-34

4. Chin KM, Kim NH, Rubin LJ. The right ventricle in pulmonary hypertension. Coron Artery Dis 2005;16:13-18.

5. Corciova FC, Arsenescu-Georgescu C. Prognostic factors in pulmonary hypertension. Maedica (Buchar) 2012;7:30-7.

6. McLaughlin VV, Archer SL, Badesch DB, et al. ACCF/AHA 2009 expert consensus document on pulmonary hypertension a report of the American College of Cardiology Foundation Task Force on Expert Consensus Documents and the American Heart Association developed in collaboration with the American College of Chest Physicians; American Thoracic Society, Inc.; and the Pulmonary Hypertension Association. J Am Coll Cardiol 2009;53:1573-619.

7. Stupi AM, Steen VD, Owens GR, et al. Pulmonary hypertension in the CREST syndrome variant of systemic sclerosis. Arthritis Rheum 1986;29:515-24.
8. Hachulla E, Gressin V, Guillevin L, et al. Early detection of pulmonary arterial hypertension in systemic sclerosis: a French nationwide prospective multicenter study. Arthritis Rheum 2005;52:3792-800.

9. Steen V, Medsger TA Jr. Predictors of isolated pulmonary hypertension in patients with systemic sclerosis and limited cutaneous involvement. Arthritis Rheum 2003;48:516-22.

10. Hoeper MM, Bogaard HJ, Condliffe R, et al. Definitions and diagnosis of pulmonary hypertension. J Am Coll Cardiol 2013;62 (Suppl 25):D42-50.

11. Gaine SP, Rubin LJ. Primary pulmonary hypertension. Lancet 1998;352:719-25.

12. Grunig E, Ehlken N, Ghofrani A, et al. Effect of exercise and respiratory training on clinical progression and survival in patients with severe chronic pulmonary hypertension. Respiration 2011;81:394-401

13. Mereles D, Ehlken N, Kreuscher S, et al. Exercise and respiratory training improve exercise capacity and quality of life in patients with severe chronic pulmonary hypertension. Circulation 2006;114:1482-9.

14. Ware JE Jr, Sherbourne CD. The MOS 36-item short-form health survey (SF-36). I. Conceptual framework and item selection. Med Care 1992;30:473-83.

15. Chia KS, Wong PK, Faux S, et al. The benefit of exercise training in pulmonary hypertension: a clinical review. Intern Med J 2016. doi:10.1111/imj.13159 [Epub ahead of print 24 Jun 2016]

16. Grunig E, Lichtblau M, Ehlken N, et al. Safety and efficacy of exercise training in various forms of pulmonary hypertension. Eur Respir J 2012;40:84-92.

17. Grunig E, Maier F, Ehlken N, et al. Exercise training in pulmonary arterial hypertension associated with connective tissue diseases. Arthritis Res Ther 2012;14:R148.

18. Nagel C, Prange F, Guth S, et al. Exercise training improves exercise capacity and quality of life in patients with inoperable or residual chronic thromboembolic pulmonary hypertension. PLOS ONE 2012;7:e41603.

19. Becker-Grunig T, Klose $\mathrm{H}$, Ehlken $\mathrm{N}$, et al. Efficacy of exercise training in pulmonary arterial hypertension associated with congenital heart disease. Int $J$ Cardiol 2013;168:375-81.

20. Ehlken $\mathrm{N}$, Lichtblau $\mathrm{M}$, Klose $\mathrm{H}$, et al. Exercise training improves peak oxygen consumption and haemodynamics in patients with severe pulmonary arterial hypertension and inoperable chronic thrombo-embolic pulmonary hypertension: a prospective, randomized, controlled trial. Eur Heart J 2016;37:35-44.

21. Ley S, Fink C, Risse F, et al. Magnetic resonance imaging to assess the effect of exercise training on pulmonary perfusion and blood flow in patients with pulmonary hypertension. Eur Radiol 2013:23:324-31.

22. Kabitz HJ, Bremer HC, Schwoerer A, et al. The combination of exercise and respiratory training improves respiratory muscle function in pulmonary hypertension. Lung 2014;192:321-8.

23. Alison Jea. The Pulmonary Rehabilitation Toolkit on behalf of the Australian Lung Foundation (2009). 2009. http://www. pulmonaryrehab.com.au.

24. Heart Foundation A. Recommended framework for cardiac rehabilitation '04. 2004. (http://www.heartfoundation.org.au/ SiteCollectionDocuments/Recommended-framework.pdf).

25. Mainguy V, Maltais F, Saey D, et al. Effects of a rehabilitation program on skeletal muscle function in idiopathic pulmonary arterial hypertension. J Cardiopulm Rehabil Prev 2010;30:319-23.

26. Fox BD, Kassirer M, Weiss I, et al. Ambulatory rehabilitation improves exercise capacity in patients with pulmonary hypertension. Jf Cardiac Failure 2011;17:196-200.

27. Chan L, Chin LM, Kennedy M, et al. Benefits of intensive treadmill exercise training on cardiorespiratory function and quality of life in patients with pulmonary hypertension. Chest 2013;143:333-43.

28. Weinstein AA, Chin LM, Keyser RE, et al. Effect of aerobic exercise training on fatigue and physical activity in patients with pulmonary arterial hypertension. Respir Med 2013;107:778-84.

29. Inagaki T, Terada J, Tanabe N, et al. Home-based pulmonary rehabilitation in patients with inoperable or residual chronic thromboembolic pulmonary hypertension: a preliminary study. Respir Investig 2014;52:357-64.

30. Saglam M, Arikan H, Vardar-Yagli N, et al. Inspiratory muscle training in pulmonary arterial hypertension. J Cardiopulm Rehabil Prev 2015;35:198-206.

31. Neuman A, Gunnbjörnsdottir M, Tunsäter A, et al. Dyspnea in relation to symptoms of anxiety and depression: a prospective population study. Respir Med 2006;100:1843-9.

32. Galiè N, Hoeper MM, Humbert M, et al. Guidelines for the diagnosis and treatment of pulmonary hypertension: the Task Force for the 
Diagnosis and Treatment of Pulmonary Hypertension of the European Society of Cardiology (ESC) and the European Respiratory Society (ERS), endorsed by the International Society of Heart and Lung Transplantation (ISHLT). Eur Heart $J$ 2009;30:2493-537.

33. Schulz KF, Altman DG, Moher D, et al. CONSORT 2010 Statement: updated guidelines for reporting parallel group randomised trials. $J$ Clin Epidemiol 2010;63:834-40.

34. Saghaei MaS S. Implementation of an open-source customizable minimization program for allocation of patients to parallel groups in clinical trials. J Biomed Sci Eng 2011;4:734-9.

35. Vonk-Noordegraaf A, Souza R. Cardiac magnetic resonance imaging: what can it add to our knowledge of the right ventricle in pulmonary arterial hypertension? Am J Cardiol 2012;110(Suppl 6):25s-31s.

36. Kellman P, Arai AE, McVeigh ER, et al. Phase-sensitive inversion recovery for detecting myocardial infarction using gadolinium-delayed hyperenhancement. Magn Reson Med 2002;47:372-83.

37. Holloway CJ, Ntusi N, Suttie J, et al. Comprehensive cardiac magnetic resonance imaging and spectroscopy reveal a high burden of myocardial disease in HIV patients. Circulation 2013;128: 814-22.

38. Holloway CJ, Montgomery HE, Murray AJ, et al. Cardiac response to hypobaric hypoxia: persistent changes in cardiac mass, function, and energy metabolism after a trek to Mt. Everest Base Camp. FASEB J 2011;25:792-6.

39. Panagiotou M, Peacock AJ, Johnson MK. Respiratory and limb muscle dysfunction in pulmonary arterial hypertension: a role for exercise training? Pulm Circ 2015;5:424-34.

40. Batt J, Ahmed SS, Correa J, et al. Skeletal muscle dysfunction in idiopathic pulmonary arterial hypertension. Am J Respir Cell Mol Biol 2014;50:74-86.

41. Breda AP, Pereira de Albuquerque AL, Jardim C, et al. Skeletal muscle abnormalities in pulmonary arterial hypertension. PLOS ONE 2014:9:e114101.

42. Dimopoulos S, Tzanis G, Manetos $\mathrm{C}$, et al. Peripheral muscle microcirculatory alterations in patients with pulmonary arterial hypertension: a pilot study. Respir Care 2013;58:2134-41.

43. Mainguy V, Maltais F, Saey D, et al. Peripheral muscle dysfunction in idiopathic pulmonary arterial hypertension. Thorax 2010;65:113-17.

44. Leong DP, Teo KK, Rangarajan S, et al. Prognostic value of grip strength: findings from the Prospective Urban Rural Epidemiology (PURE) study. Lancet 2015;386:266-73.

45. Pandey A, Garg S, Khunger M, et al. Efficacy and safety of exercise training in chronic pulmonary hypertension: systematic review and meta-analysis. Circ Heart Fail 2015;8:1032-43.

46. Buys R, Avila A, Cornelissen VA. Exercise training improves physical fitness in patients with pulmonary arterial hypertension: a systematic review and meta-analysis of controlled trials. BMC Pulm Med 2015;15:40.

47. Holland AE, Spruit MA, Troosters T, et al. An official European respiratory society/American thoracic society technical standard: field walking tests in chronic respiratory disease. Eur Respir $J$ 2014:44:1428-46.

48. Löwe B, Gräfe K, Ufer C, et al. Anxiety and depression in patients with pulmonary hypertension. Psychosom Med 2004;66:831-6.

49. Harzheim D, Klose H, Pinado FP, et al. Anxiety and depression disorders in patients with pulmonary arterial hypertension and chronic thromboembolic pulmonary hypertension. Respir Res 2013;14:104.

50. Shafazand S, Goldstein MK, Doyle RL, et al. Health-related quality of life in patients with pulmonary arterial hypertension. Chest 2004;126:1452-9.

51. Graf $C$. The Lawton instrumental activities of daily living scale. Am J Nurs 2008;108:52-62; quiz 62-3.

52. McKenna SP, Doughty N, Meads DM, et al. The Cambridge Pulmonary Hypertension Outcome Review (CAMPHOR): a measure of health-related quality of life and quality of life for patients with pulmonary hypertension. Qual Life Res 2006;15:103-15.

53. Ganderton L, Jenkins S, McKenna SP, et al. Validation of the Cambridge Pulmonary Hypertension Outcome Review (CAMPHOR) for the Australian and New Zealand population. Respirology 2011;16:1235-40.

54. Graf C. The Lawton Instrumental Activities of Daily Living (IADL) Scale. Medsurg Nurs 2009;18:315-16.

55. Henry JD, Crawford JR. The short-form version of the Depression Anxiety Stress Scales (DASS-21): construct validity and normative data in a large non-clinical sample. Br J Clin Psychol 2005;44(Pt 2):227-39.

56. Nagaya N, Nishikimi T, Uematsu $\mathrm{M}$, et al. Plasma brain natriuretic peptide as a prognostic indicator in patients with primary pulmonary hypertension. Circulation 2000;102:865-70.

57. Heresi GA, Aytekin M, Hammel JP, et al. Plasma interleukin-6 adds prognostic information in pulmonary arterial hypertension. Eur Respir $J$ 2014;43:912-14.

58. Souza R, Jardim C, Julio Cesar Fernandes C, et al. NT-proBNP as a tool to stratify disease severity in pulmonary arterial hypertension. Respir Med 2007;101:69-75.

59. Fijalkowska A, Kurzyna M, Torbicki A, et al. Serum N-terminal brain natriuretic peptide as a prognostic parameter in patients with pulmonary hypertension. Chest 2006;129:1313-21.

60. de Man FS, van Hees HW, Handoko ML, et al. Diaphragm muscle fiber weakness in pulmonary hypertension. Am J Respir Crit Care Med 2011:183:1411-18.

61. Kabitz HJ, Schwoerer A, Bremer HC, et al. Impairment of respiratory muscle function in pulmonary hypertension. Clin $\mathrm{Sci}$ 2008;114:165-71.

62. American Thoracic Society/European Respiratory Society. ATS/ERS Statement on respiratory muscle testing. Am J Respir Crit Care Med 2002;166:518-624.

63. Scherr J, Wolfarth B, Christle JW, et al. Associations between Borg's rating of perceived exertion and physiological measures of exercise intensity. Eur J Appl Physiol 2013;113:147-55.

64. Holland AE, Wadell K, Spruit MA. How to adapt the pulmonary rehabilitation programme to patients with chronic respiratory disease other than COPD. Eur Respir Rev 2013;22:577-86.

65. Spruit MA, Singh SJ, Garvey C, et al. An official American Thoracic Society/European respiratory society statement: key concepts and advances in pulmonary rehabilitation. Am J Respir Crit Care Med 2013; 188:e13-64.

66. Hill K, Cecins NM, Eastwood PR, et al. Inspiratory muscle training for patients with chronic obstructive pulmonary disease: a practical guide for clinicians. Arch Phys Med Rehabil 2010;91:1466-70.

67. Cahalin LP, Arena RA. Breathing exercises and inspiratory muscle training in heart failure. Heart Fail Clin 2015;11:149-72.

68. Kraemer WJ, Adams K, Cafarelli E, et al. American college of sports medicine position stand. Progression models in resistance training for healthy adults. Med Sci Sports Exerc 2002;34:364-80.

69. Gloeckl R, Marinov B, Pitta F. Practical recommendations for exercise training in patients with COPD. Eur Respir Rev 2013:22:178-86.

70. CRUFAD CRUFAD, St Vincent's Hospital Sydney, Australia. Anxiety and Panic Disorder. Patient Treatment Manual. 2010. https://crufad. org/images/stories/pdf/manuals/crufad_Panicmanual.pdf (accessed 14 Mar 2016).

71. Sage N, Sowden M, Chorlton E, et al. CBT for chronic illness and palliative care: a workbook and toolkit. England: John Wiley and Sons, 2008

72. Jacobsen E. Progressive relaxation. Chicago, IL, USA: University of Chicago Press, 1938

73. NHMRC. National Statement on Ethical Conduct in Human Research (2007)—Updated March 2014. 2007.

74. Kawut SM, Horn EM, Berekashvili KK, et al. New predictors of outcome in idiopathic pulmonary arterial hypertension. Am J Cardiol 2005;95:199-203.

75. Addetia K, Bhave NM, Tabit CE, et al. Sample size and cost analysis for pulmonary arterial hypertension drug trials using various imaging modalities to assess right ventricular size and function end points. Circ Cardiovasc Imaging 2014;7:115-24.

76. Bradlow WM, Hughes ML, Keenan NG, et al. Measuring the heart in pulmonary arterial hypertension (PAH): implications for trial study size. J Magn Reson Imaging 2010;31:117-24.

77. Zhong B. How to calculate sample size in randomized controlled trial? J Thorac Dis 2009;1:51-4. 